\title{
Emotional Regulation in Mothers and Fathers and Relations to Aggression in Hong Kong Preschool Children
}

\author{
Eva Yi Hung Lau ${ }^{1}(1) \cdot$ Kate Williams ${ }^{2}$
}

Accepted: 21 March 2021 / Published online: 12 April 2021

(c) The Author(s) 2021

\begin{abstract}
This study explored the associations among emotional regulation in mothers and fathers and preschool children's physical and relational aggression using a Hong Kong Chinese sample. This study also explored whether child gender would moderate the association between parental emotional regulation strategies and children's physical and relational aggression. Participants were 168 children aged 4-6 years. Parents reported on their own emotional regulation approaches and kindergarten class teachers rated children's aggression 6 months later. Path analyses showed that higher levels of reappraisal and lower levels of suppression by mothers was associated with higher levels of child relational aggression. There were no significant associations among fathers' emotional regulation and children's aggression. Results from multi-group analysis showed that there were no significant moderation of the associations by child gender. Results highlight the importance of mothers' emotional regulation in child aggression and suggest that the maladaptive consequences of emotional suppression are culturally relative.
\end{abstract}

Keywords Reappraisal $\cdot$ Suppression $\cdot$ Aggression $\cdot$ Child gender $\cdot$ Hong Kong Chinese

\section{Introduction}

Aggression, defined as behavior intended to harm others, is one of the most common types of childhood behavioral problems [1,2]. Physical and relational aggression are the two most common forms of aggression used by children during the early childhood years [3]. Whereas physical aggression involves harming or threatening harm by means of physical damage (e.g., hitting and pushing), relational aggression is defined as harm or the threat of damage that is intended to manipulate or damage peer relationships (e.g. spreading rumors and threats to withdraw friendship) [4]. Aggressive children have been found to have high levels of psychological distress, low levels of prosocial behavior, and high levels of peer rejection [5]. Because of the numerous difficulties experienced by aggressive children, antecedents of childhood aggression are crucial to understand for developing prevention and intervention programs.

Eva Yi Hung Lau

evalau@eduhk.hk

1 Department of Early Childhood Education, The Education University of Hong Kong, Hong Kong, Hong Kong SAR

2 School of Early Childhood and Inclusive Education, Queensland University of Technology, Brisbane, Australia
Parenting and family experiences are a key context in which children's socio-emotional behaviors develop [6-9]. The parenting environment is especially influential during the preschool years when children are most open to parental influence [10]. In particular, studies have identified parental emotional regulation approaches as an important influencing factor for child behaviors and developmental outcomes [11-13]. Although there has been an increasing number of studies in the area of mothers' emotional regulation and children's externalizing behaviours [14-16], they are limited in their cross-sectional design, the lack of examination of fathers' influence, and lack of representation of nonWestern samples. To the best of our knowledge, no studies have investigated the precise role of parental emotional regulation in the development of children's physical and relational aggression. This study explores associations of mothers' and fathers' emotional regulation and the use of physical and relational aggression by young children using a Chinese sample. This study will inform the understanding of how parental emotional regulation among Chinese parents affects child's physical and relational aggression for developing practices to prevent or intervene children's aggression through parental emotional regulation.

Emotional regulation refers to the processes responsible for monitoring, evaluating, and modifying emotional 
reactions to accomplish one's goals [17]. These skills are considered a cornerstone of psychosocial functioning, with poorer emotional regulation implicated in many health and mental disorders $[18,19]$. Two specific emotional regulation strategies in adults are commonly conceptualised and measured. Cognitive reappraisal refers to the ability to change thoughts associated with an emotion-inducing event in such a way as to modify its emotional impact [20]. Reappraisal is generally considered a highly adaptive emotional regulation approach associated with more positive psychosocial outcomes [21]. Emotional suppression refers to a strategy whereby individuals refrain from acknowledging or expressing emotions in an effort to regulate them [20] and is typically considered a maladaptive approach associated with poorer psychosocial outcomes over time [21].

Although parental emotional regulation is suggested to be important in shaping how parents act as emotion socialization agents for their children, it remains an understudied topic $[22,23]$. Numerous studies have demonstrated the links between parents' self-regulation and children's outcomes [24-26]. However, only a few studies [14-16] have examined the links between parental use of emotional regulation strategies and children's social behaviours, such as externalizing (e.g., impulsivity and hyperactivity) and prosocial (e.g., helping and sharing) behaviour, with none addressing childhood aggression specifically. In general, these studies find that higher use of adaptive emotional regulation approaches, including reappraisal, and lower use of maladaptive emotional regulation strategies, including suppression, by parents is associated with more positive social-emotional development in children. For instance, Crespo and colleagues [14] studied 454 American mothers and their children aged 3-7-years-old. These researchers found that children's difficulties with emotional regulation mediated the relation between mothers' lower emotion awareness and both internalizing and externalizing, whereas there was no significant direct associations between maternal difficulties with emotional regulation and children's externalising behaviour problems, Specifically, maternal difficulties with emotional regulation were associated with higher levels of emotional negativity in children which in turn was associated with more externalizing behaviour problems in children. In an intervention study with 139 Australian children aged 29-83 months and their caregivers (93\% mothers) referred for parenting support, it was found that increased use of cognitive reappraisal by caregivers was associated with a decline in children's externalizing behavior problems [16]. A further recent study documented that higher levels of emotional suppression in caregivers (86\% mothers) was associated with fewer prosocial behaviours in 3-year-old children in the US, with reappraisal by caregivers having no significant association [15].
In relation to childhood aggression specifically, parental adaptive emotional regulation, characterised by high levels of reappraisal and low levels of suppression, might reduce a child's use of aggression through modelling processes and an influence on parenting behaviors. First, children may observe and imitate their parents' emotional regulation capacities [27, 28]. It is possible that parents' own emotional profiles and interactions implicitly teach children which emotions are acceptable and expected in the family environment, and how to manage the experience of those emotions [11]. It is also possible that parental emotional regulation creates a family emotional climate through which children will learn the appropriateness of emotional displays [29, 30]. As a result, parents' modelling of poor emotional regulation approaches and children's social references of these regulation strategies may contribute to children's poor regulation. In fact, children with behavior problems have consistently been found to have limited emotion understanding, to display more negative emotions, and to have poorer capacity to regulate these emotions [31, 32].

Second, parents' emotional regulation will affect their parenting practices. Parenting often involves numerous challenges and demands that require parents to remain calm and maintain positive affect in order to set clear limits for child behaviour and consistently follow through on stated rules. For parents with adaptive emotional regulation, they may be more responsive to their children's emotional needs and emphasize empathic goals and problem solving strategies in the processes of socialization, which nurture their children to regulate negative emotions and undesirable tendencies that may result in aggression [33-35]. On the other hand, parents with poorer emotional regulation capacities may show higher levels of hostility and rejection and lower levels of emotional availability, which creates a context that is less supportive of children's emotional and behavioural adjustment [24, 26, 36, 37].

In addition to a notable lack of research on the role of parental emotional regulation strategy use and children's relational and physical aggression overall, there is a distinct lack of examination of mothers vs fathers and moderation of any effects by child gender. Given the complexity of family dynamics, delineating effects by parent and child gender is crucial in child and family studies. Traditionally, Chinese fathers have been regarded as the strict parent who is responsible for disciplining children in the family, with their general involvement in other aspects of parenting considered lower than mothers' [38]. However, because of modernization and westernization, families in China, including Hong Kong, have undergone numerous changes in more recent years. For instance, gendered parenting roles have become less pervasive as most Hong Kong women aspire to obtain higher education and achieve an active working life [39, 40]. As a result, Chinese fathers are becoming more involved in 
child rearing and are considered important socializing agents in children's development [40-42].

The role of fathers' emotional regulation strategies in children's social emotional development, particularly in aggression, has been largely unaddressed in the research literature to date. Contemporary fathers, including Chinese fathers, aspire to be actively involved in their children's development [42]. Studies have also documented the positive influence of fathering on child developmental outcomes [43] and have shown a significant relation between fathers' ER and their parenting practices [44]. Hence, understanding how fathers' parenting can be promoted from infancy by facilitating prenatal ER is considered critical.

Available evidence from work examining fathers' parenting and self-regulatory approaches more generally yields mixed findings. Some studies suggest that fathers' behaviors exert a stronger influence than mothers'. For example, compared to mother-infant interactions, father-infant interactions have had stronger associations with infants' [45] (Portugal) and toddlers' emotional regulation [46] (United States); and paternal, but not maternal psychological aggression predicted children's emotional regulation in middle childhood [47] (China). Still other studies suggest that fathers exert an equal influence to mothers. For example, fathers' emotional dysregulation was found to be as likely to influence levels of parent-child aggression in families with pre-schoolers in the United States as mothers' dysregulation [48]. It is clear that more research is needed to better establish the role of fathers' emotional regulation.

Child gender is also an important consideration and may moderate relations among parenting and children's socialemotional development. Previous studies have suggested that boys and girls differed in their response to maternal emotional expression $[49,50]$. Some literature suggests that parents are more likely to socialize daughters' emotional and social development than sons, and that girls are more sensitive to family affective environment than boys [11, 51]. However, other research has documented that both mothers and fathers used more emotional language with boys than with girls [52], and lower levels of maternal sensitivity have been found to be particularly detrimental to boys in terms of their development of externalizing behavior problems [53]. It is clear that further research on the role of child gender in the context of emotional regulation by both mothers and fathers is warranted.

Differences in cultural and parenting norms are likely to influence parents' emotional regulation approaches and the ways in which they influence children's social-emotional development. While most research to date on parents' emotional regulation and children's social-emotional functioning has been done with Western participants, there is a growing field of research with Chinese participants and other collectivist cultures. However, these studies have yielded mixed results. On one hand, it is possible that although suppression has typically been found to be maladaptive, the maladaptive consequences of suppression may be culturally relative. Specifically, among collectivist cultures, freely expressed emotions, particularly negative emotions, are often discouraged as they are thought to disrupt group cohesiveness and social harmony [54]. For example, Butler, Lee and Gross [55] and Cheung and Park [56] found that suppressers who held Western-European values had poorer social and emotional outcomes during interpersonal interactions than did suppressers who held Asian values. Similarly, Yuan et al. [57] and Soto et al. [58] found that suppression may be more adaptive among participants in the Chinese context than in Western cultures. In contrast, other studies have identified the costs associated with suppressing with Chinese and Chinese American samples, including psychological distress, social functioning difficulties, and health issues [59, 60]. Given the limited existing evidence base and the todate mixed findings, the present study is necessary to better understand the associations between parental emotional regulation and child aggression within the Chinese context.

Researchers have increasingly realized the importance of the fathers' role in children's development [61-63]. However, relatively less studies have examined how fathers' emotional regulation strategies may be associated with children's aggression and even fewer studies have been conducted in a Chinese context. The present study used a Hong Kong Chinese sample to explore the direct relations between both mothers' and fathers' emotional regulation and child aggression. This study also explored whether the association between mothers' and fathers' emotional regulation and child aggression would be moderated by child gender. Based on previous work, it was hypothesized that higher use of reappraisal by both mothers and fathers would be related to lower physical and relational aggression in children. Because of the cultural context, we hypothesized that higher levels of suppression used by mothers and fathers would also be related to lower physical and relational aggression in children, in our study conducted in a collectivist culture. Given the mixed findings to date about the role of fathers and the effect of child gender, we had no specific hypotheses about the nature and strength of father associations compared to mother associations on boys versus girls.

\section{Method}

\section{Participants and Procedure}

In the present study, the participants were recruited using a stratified and convenience sampling strategy. Specifically, the number of classes invited in each of the three main territories, namely Hong Kong Island, Kowloon, and New 
Territories, varied so that the percentages of participants from each territory resembled the percentages of the Hong Kong population in each area. In 2016, approximately 17.1, 30.6 and $52.3 \%$ of the Hong Kong population lived in Hong Kong Island, Kowloon, and New Territories, respectively [64]. All kindergartens in Hong Kong are privately run and provide services for children from three to six years old. Nearly all children in Hong Kong start kindergarten at age three and they are grouped into classes according to their age (e.g., 3-4-year-old, 4-5-year-old, and 5-6-year-old). Invitation letters were sent to kindergartens and phone calls were made to the principals. Five kindergartens (two from each of Hong Kong Island and New Territories and one from Kowloon) agreed to participate in the study. A total of 17 classes from the 5 kindergartens were invited to participate. All teachers ( $\mathrm{N}=17 ; 16$ females) of the participating classes consented to participation.

The teachers distributed introductory letters about the study to all parents in their class. A total of 175 parents provided consent to participate in this study (Hong Kong Island: 18.3\%, Kowloon: $34.3 \%$, and New Territories: $47.4 \%$ ). Unfortunately, the consent rate was not available because some of the kindergartens failed to provide information about their class sizes. Seven of the children were excluded due to their special educational need (e.g. social functioning and cognitive functioning). The final sample included a total of 168 children from five $4-5$-year-old classes $(N=35)$ and $125-6$-year-old classes $(N=133)$. The children (52\% girls) were on average 61 months old ( $S D=5.51$ months).

The mothers and fathers of the children were on average 35.6 years $(S D=4.93)$ and 39.9 years $(S D=6.12)$ old respectively. The median education level that the participating parents had attained was secondary education $(58.9 \%$ of mothers and $55.4 \%$ of fathers), which was also the median education level of the Hong Kong population in 2016 [64]. In terms of marital status, 149 sets of parents reported themselves as married, five sets of parents as divorced, one set of parents remarried and six sets of parents selected "others" as their marital status (i.e. single or separating). The median monthly household income of the participating families was $\operatorname{HK} \$ 27,000(M=29,450 ; S D=18,713 ; \mathrm{US} \$ 1=\mathrm{HK} \$ 7.78)$, which was slightly higher than the median monthly household income of Hong Kong families (HK\$24,890) [64].

In this study, data were collected at two time points with a six-month interval in order to examine the predictive effect of parent emotion regulation (Time 1 predictor) on subsequent child aggression (Time 2 outcome) between individuals. The six-month interval in between Time 1 and Time 2 data collection would ensure sufficient time for the child behaviors to develop within the project timeframe for testing the hypothesized relations. At Time 1, approximately 3 months after the school year has started, we invited parents to complete questionnaires related to their family background and their own emotional regulation. Among the 168 families, 151 (90\%) of them returned both father and mother questionnaires, seven families only returned the mother questionnaire, three families only returned the father questionnaire, and seven families failed to return any parent questionnaires. At Time 2, approximately 6 months after the first time point, the lead teacher in each participating class was invited to provide ratings of physical and relational aggression for each participating child, except one child who changed school after Time 1. Completed questionnaires $(N=167)$ were successfully obtained from all participating teachers. Each family received a supermarket coupon at the value of USD6 as a token of appreciation for their participation.

\section{Measures}

\section{Parental Emotional Regulation}

Parents' emotional regulation was assessed with the Emotion Regulation Questionnaire [65]. Each parent reported their own emotion regulatory processes using the 6-item reappraisal subscale (e.g., "I control my emotions by changing the way I think about the situation I am in") and 4-item suppression subscale (e.g., "I keep my emotions to myself") subscale. Each item was rated on 7-point scale $(1=$ strongly disagree, $7=$ strongly agree $)$. In the present study, the two subscales achieved adequate internal consistency for both mothers (reappraisal: 0.85; suppression: 0.74 ) and fathers (reappraisal: 0.88; suppression: 0.75). The scores for reappraisal and suppression were generated by averaging the items within each subscale. Higher scores indicated higher levels of reappraisal and suppression.

\section{Child Aggression}

The Preschool Social Behavior Scale [66] was used to assess teachers' rating of children's physical aggression (8 items; e.g., "This child pushes or shoves other children") and relational aggression (9 items; e.g., "This child tells others not to play with or be a peer's friend") on a fivepoint Likert scale $(1=$ never; $5=$ always $)$. Similar to previous studies involving Chinese populations (e.g., [42]), the two subscales showed strong internal consistency (physical aggression: 0.92, and relational aggression: 0.94) in this study. The scores for physical aggression and relational aggression were generated by averaging the items within each subscale. Higher scores indicated higher levels of aggression. 


\section{Approach to Analysis}

All analyses were conducted in Mplus Version 7.11 [67]. We first tested a whole sample model followed by a multigroup model that produced separate path estimates for boys and girls and tested sequentially constrained models to compare model fit using BIC. Missing data was minimal ranging from zero missing data on child aggression variables to $8.3 \%$ missing data for fathers' emotional regulation. Missing data was handled through use of maximum likelihood estimation within the models.

It is important to note that outcome data for teacherreported children's aggression is nested within classrooms. Intra class correlations for teacher reported aggression were 0.26 and 0.31 for physical and relational aggression respectively, creating corresponding variance inflation factors of 3.27 and 3.73. However, due to sample size, the model is underpowered to account for clustering in estimation. This increases the chance of Type 1 error and is acknowledged as a limitation of the study. Results should therefore be interpreted with caution and considered exploratory until results are produced by replication studies with larger sample sizes or non-clustered outcome data.

\section{Results}

\section{Descriptive Statistics}

Bivariate correlations among all variables are provided in Table 1. Children's physical and relational aggression as reported by teachers was strongly correlated. Boys displayed more physical aggression but gender was not correlated with relational aggression. Being a mother of a boy and a more highly educated mother was associated with less use of suppression. Mother's use of reappraisal showed small but significant correlations with mothers' use of suppression and fathers' use of reappraisal. Father's reappraisal and suppression emotional regulation were also moderately correlated.

\section{Mothers' and Fathers' Emotional Regulation and Children's Physical and Relational Aggression}

The path analysis for the associations among mothers' and father's self-reported emotional regulation approaches and children's teacher-reported aggression, was a good fit to the data (Fig. 1). Higher levels of reappraisal and lower levels of suppression by mothers was associated with higher levels of relational aggression in children. There was a marginal association with higher levels of mothers' reappraisal associated with higher levels of children's physical aggression. There were no associations between fathers' emotional regulation strategies and children's aggression. Because this model controlled for mothers' emotional regulation, we also tested a model that included only fathers' self-regulation in relation to children's aggression. No significant associations among fathers' emotional regulation and children's aggression were found in this independent model.

\section{Child Gender as a Moderator}

The multi-group analysis for boys and girls was also a good fit to the data (Fig. 2). However, constraining paths to be equal across gender did not worsen model fit as assessed by BIC, thus there is no evidence that there is statistically significant moderation of associations by gender. However, interpretation of the estimates yields some interesting points. In this model the associations between mothers' reappraisal and higher child relational aggression, and mothers' suppression and lower child relational

Table 1 Bivariate correlations among all variables modelled

\begin{tabular}{|c|c|c|c|c|c|c|c|c|c|c|}
\hline & & 1 & 2 & 3 & 4 & 5 & 6 & 7 & 8 & 9 \\
\hline 1 & Boy & 1 & & & & & & & & \\
\hline 2 & Age (months) & -.06 & 1 & & & & & & & \\
\hline 3 & Maternal education & .09 & .06 & 1 & & & & & & \\
\hline 4 & Mothers' reappraisal & .08 & -.04 & .07 & 1 & & & & & \\
\hline 5 & Mothers' suppression & $-.16 *$ & .05 & $-.17 *$ & $.21 *$ & 1 & & & & \\
\hline 6 & Fathers' reappraisal & -.02 & .04 & .02 & $.20^{*}$ & -.05 & 1 & & & \\
\hline 7 & Fathers' suppression & -.06 & -.03 & -.11 & -.02 & .03 & $.41 * *$ & 1 & & \\
\hline 8 & Child physical aggression & $.25^{*}$ & .10 & -.04 & .08 & -.06 & -.06 & -.01 & 1 & \\
\hline \multirow[t]{5}{*}{9} & Child relational aggression & -.03 & .08 & -.04 & .11 & -.12 & -.01 & .01 & $.74 * *$ & 1 \\
\hline & Range & & $46-71$ & $1-6$ & $2-7$ & $1-6.33$ & $1-7$ & $1-6.75$ & $1-3.38$ & $1-3.89$ \\
\hline & Mean & & 60.97 & 2.63 & 5.03 & 3.82 & 4.93 & 4.19 & 1.29 & 1.62 \\
\hline & $S D$ & & 5.51 & .92 & .92 & 1.07 & 1.03 & 1.09 & .51 & .71 \\
\hline & $\alpha$ & & & & .85 & .74 & .88 & .75 & .92 & .94 \\
\hline
\end{tabular}

$* p<.05 ; * * p<.01$ 


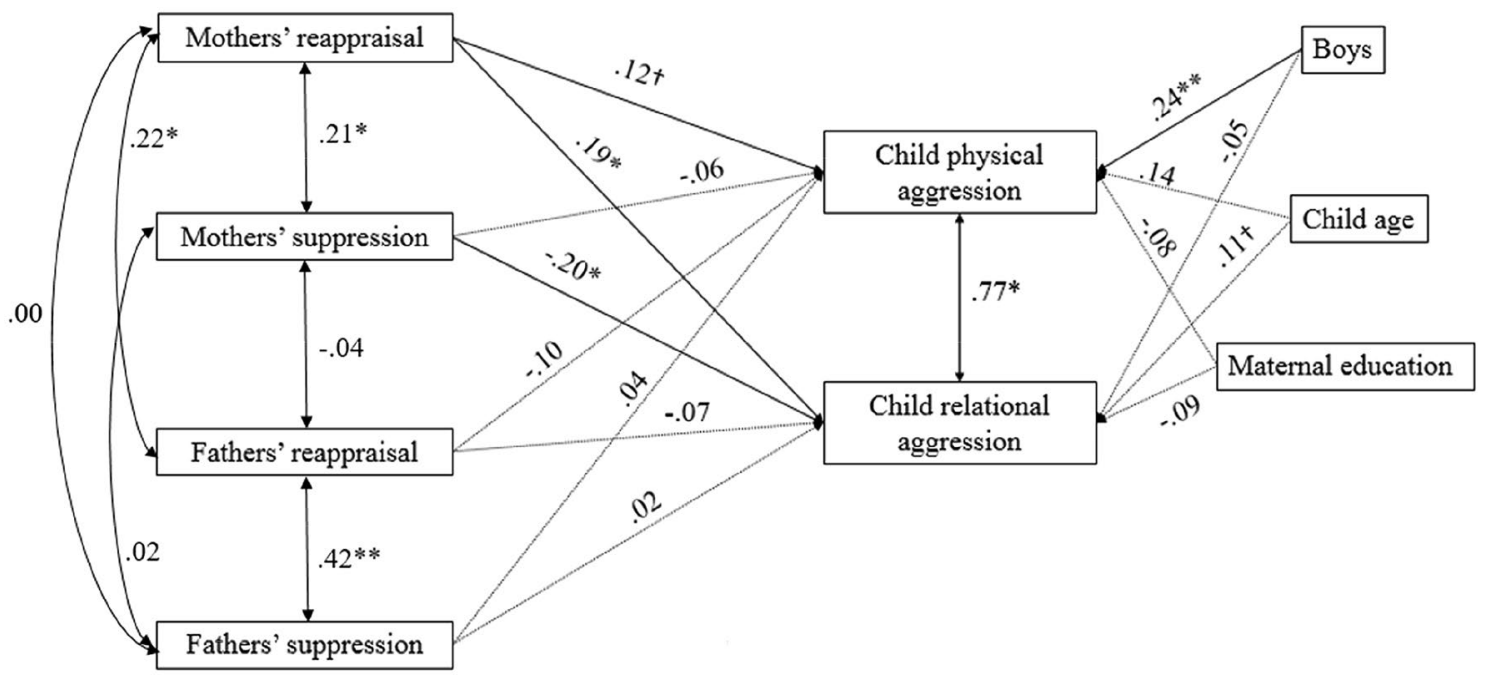

Fig. 1 Path model for the effect of early mothers' and fathers' selfreported emotional regulation strategies on later physical and relational aggression in children, as reported by teachers. Fit indices: $x^{2}=16.81, d f=12, p=.16$; RMSEA $=.05, \mathrm{CI}=.00$ to $.10 ; \mathrm{CFI}=.97$, $\mathrm{BIC}=2297.683$. Dotted lines indicate non-significant path estimates. ${ }^{\dagger} p<.10 ; * p<.05 ; * * p<.01$. All estimates are standardized

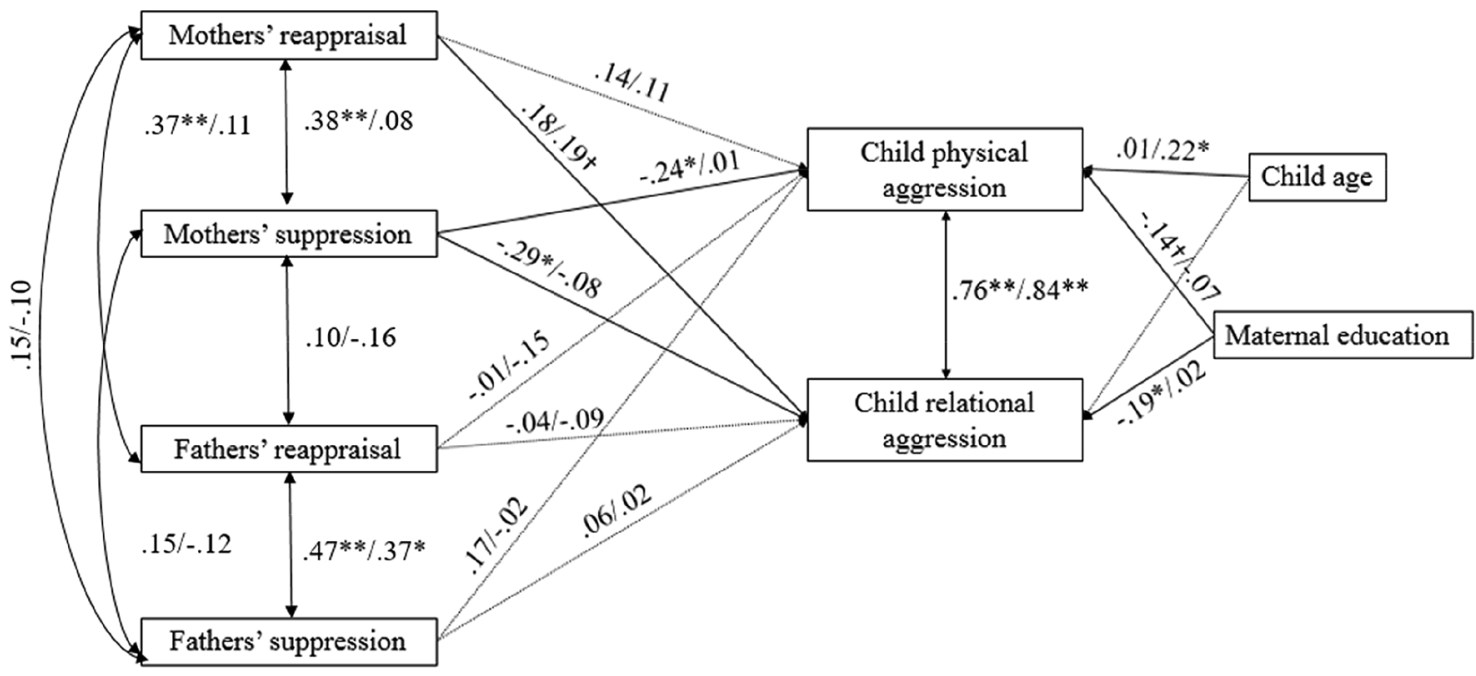

Fig. 2 Path model for the effect of early mothers' and fathers' selfreported emotional regulation strategies on later physical and relational aggression in children, as reported by teachers. Fit indices: $x^{2}=10.86, d f=16, p=.81 ; \mathrm{RMSEA}=.00, \mathrm{CI}=.00$ to $.64 ; \mathrm{CFI}=1.00$,

aggression remained. The association at the whole group level between higher use of suppression by mothers and lower levels of child relational aggression appeared to be driven primarily by an influence on girls in particular. This influence of mothers on girls was also reflected in a new association that did not appear in the whole sample model, with mothers' use of suppression being related to lower levels of physical aggression in children, particularly for girls.
$\mathrm{BIC}=2359.30 .{ }^{\dagger} p<.10 ; * p<.05 ; * * p<.01$. All estimates are standardized. Paired parameters indicated standardized estimates for girls/ boys. Differences across gender are not significant 
of parental emotional regulation on children's aggression using a Chinese Hong Kong sample. Consistent with existing studies [14-16], the present study found that parents' emotional regulation is directly relevant to children's social behaviors. More specifically, we found that maternal, but not paternal, emotional regulation was related to child aggression. Such findings are consistent with previous research suggesting that mothers have a stronger influence on child socioemotional outcomes because they are more likely to be involved in the development of children's ability to cope or manage their emotional expression and experience $[51,69,70]$. A number of implications for future research and practice are discussed below.

Contrary to our hypothesis, maternal reappraisal was found to predict higher levels of physical and relational aggression. Cognitive reappraisal involves changing thoughts associated with an emotion-inducing event that has been acknowledged [20]. For example, a parent who is experiencing a stressful work event may use cognitive reappraisal to regulate the induced negative emotions by acknowledging and accepting her negative emotions, followed by an evaluation of the benefits or opportunities that the event may bring. These processes are important prerequisite to the execution of a solution. Compared to that of emotional suppression, the the internal nature of cognitive reappraisal is more complex and may not provide a context in which this regulatory approach could be learned [6]. Socialization involves parents' direct and indirect influences on children's experience as well as regulation of emotions and behaviours through parents' own expression of emotion, responsiveness, and guidance [22, 71-73]. Through observing their mothers' use of reappraisal in regulating their own emotions, children may learn to be aware and accept their own emotions indirectly, but not necessarily learn to manage their emotions through the execution of a solution effectively. As a result, a lack of direct guidance and instruction (e.g., emotion coaching) from the parent to the child in a healthy, age-appropriate way may leave children with fewer strategies for managing their negative emotion or solving peer conflicts, in which they may use more aggression to express the negative emotions induced by their interpersonal problems that they have identified and experienced. The above findings highlight the importance of providing trainings for parents and children so that they can both learn to use effective emotional regulation strategies directly. Clinicians and parent educators can also coach parents to teach children how to manage their emotions through the execution of a solution to resolve peer conflicts, in addition to only identifying and being aware of their negative emotions. As parents' reappraisal processes may not be explicit and verbalised, parents should also be supported to verbalise the emotional regulation strategies they use so that they can influence children's own emotional regulation and aggression.
Consistent with existing studies that the maladaptive consequences of suppression may be culturally relative and that suppression is not related to externalizing behaviors such as aggression in collectivist cultures [55-58], we found evidence supporting our hypothesis that higher levels of maternal suppression predicted lower relational aggression in children. As freely expressed emotion, particularly negative emotions, is often discouraged for maintaining group cohesiveness in the Chinese context [54], mothers may try to protect their children from experiencing adverse responses by hiding their feelings from their children. As a result, the use of suppression may be somewhat normalized and accepted by children in Hong Kong. Specifically, children may learn by observing and interacting with their mothers that suppressing their emotions and refraining from acting in ways that would disrupt social harmony is appropriate and hence, reduces children's use of relational aggression. Although suppression was found to decrease children's relational aggression as a negative expression, numerous studies have suggested the use of suppression as a risk factor for internalizing problems such as increased depressive and anxiety symptoms in both Western $[74,75]$ and Asian collectivist cultures [59]. It has also been suggested that suppression may hinder parents' ability to respond sensitively and appropriately to children's emotions, which can be particularly damaging in the context of parent-child relationships [76]. Therefore, more studies investigating parental suppression, child suppression, child aggression and child internalizing problems in the Chinese context are needed to better understand the nature and influences of suppression in diverse cultures. Nevertheless, the findings suggested that parents with low levels of suppression in the Chinese culture may be a risk factor for children's aggressive behaviors, clinicians and parent educators should encourage a balance between appropriate parental emotional expression and suppression.

In this study, neither the model with both fathers and mothers included, or an independent model with only fathers, yielded significant associations between paternal emotional regulation strategies and children's aggression. The finding is in contrast to more general studies on the role of fathers in children's social-emotional development which suggest an at-least-equal-to, if not greater, influence as mothers [45-47]. However, most prior studies have been conducted with Western samples and none, to our knowledge, have specifically examined paternal emotional regulation and childhood aggression. It is likely that our findings reflect a context in which mothers are the main caretakers of children and thus more likely to be involved in the socialization of children's emotional and social behaviors. As such, it is possible that the level of paternal involvement would moderate the relation between paternal emotional regulation and child aggression, in which fathers' emotional regulation would only influence child aggression when they are highly 
involved in their development. Nevertheless, as role expectations for mothers and fathers continue to change in Chinese societies, further research is required to replicate these findings and further understand potential mediators of these associations. For example, while paternal emotional regulation was not a significant influence in the current study, it may have an important influence on children's aggressive behaviors through externalized parenting acts by fathers including expressed warmth, hostility, and engagement in home learning activities. Importantly, evidence produced by the current study, along with others, can be used to inform the target audience and behaviors of parenting support programs that aim to influence child developmental outcomes. For example, differentiated interventions might be needed for mothers vs fathers, with different targets for knowledge and behavior change.

While there was no statistically significant evidence of moderation by child gender in this study, there was a trend toward stronger associations between maternal emotional regulation and girls' aggression, which may have reached significance with a larger sample size. In particular, mothers' use of suppression was associated with lower levels of both relational and physical aggression for girls. Girls may be particularly susceptible to the socialization of suppression as they use their mothers as role models to guide behavior. As discussed above, suppression in the collectivist culture may act to reduce overt aggression and maintain social cohesion, and this may be particularly so for girls. This finding reflects prior research that suggests girls are more sensitive to mothers' emotional expression and parenting approaches than boys $[50,77]$. Further research is needed to develop a more comprehensive understanding of a range of parental influences in relation to the development of childhood aggression. The implications of differential associations for boys and girls are that different intervention targets and approaches for boys and girls might be needed to intervene on or prevent aggressive behaviors.

\section{Limitations and Future Directions}

This study provides initial evidence suggesting that parental use of emotional regulation strategies may be an important influence on child aggression. Our findings add to the growing body of literature indicating that aspects of parental self-regulation are related to important child outcomes and influence the context in which children are being raised. This study exhibited several notable strengths such as the collection of data at two time points and the inclusion of both mothers and fathers. Nevertheless, several limitations of this study must be acknowledged. First, this study was limited by the examination of the direct associations between parental emotional regulation strategies and forms of child aggression, without the inclusion of potential mediators such as the quantity of parental involvement and quality of parenting behaviors. In practice, it is likely that parental emotional regulation does not directly influence how aggressively children behave with peers, but instead influences this more distal outcome by altering parenting strategies and children's socioemotional functioning [78]. In particular, the unexpected findings of the positive relations between maternal reappraisal and child physical and relational aggression could be better articulated by including variables such as parents' emotion coaching strategies. Similarly, while paternal emotional regulation was not related to any child outcomes, the importance of the paternal emotion variables for children's aggression might become more apparent if parenting behavior or children's emotional regulation is included as the mediating mechanism. It is also possible that fathers' emotional regulation would only have an impact on child aggression when they are highly involved. Hence, future research with a focus on assessing these potential mechanisms is needed to examine such complex, multiple mediated and moderated effects. Second, parental self-reports of emotional regulation may be different in a setting that induces strong affect as opposed to the general context in which it was assessed in the current study. Therefore, the conclusions that were drawn from this study regarding the processes by which parental emotional regulation contributes to child aggression should be interpreted with caution. Future studies should assess parental emotional regulation during parenting as it is the context in which children are likely to be affected by their parents' emotional regulation strategy use. Third, as in previous studies in both Western [79] and non-Western [80] contexts, our study involved a sample of pre-schoolers who displayed relatively low levels of aggression. The results may be different when examining children with more elevated aggression scores. Relatedly, we only used teacher reports for assessing child aggression. Future studies should consider replicating this study within a more clinical sample and using a multi-informant approach or utilize observation methods to obtain data of child aggression. Fourth, our ability to detect potentially small but important effects was limited by our relatively small sample size. Fifth, with children nested within schools and within classrooms, the possible non-independence of teacher ratings of child aggression should be noted. Hence, results should be considered exploratory and interpreted with caution. Future studies should consider replicating this study by employing larger samples or non-clustered outcome data to examine similar questions. Sixth, finally, as Hong Kong is considered the most westernized city in China, our findings may not be generalized to parents in Mainland China. Future studies should be conducted in other areas in Mainland China before the findings are generalized. 


\section{Summary}

This study is the first, to our knowledge, to document the associations between maternal and paternal emotional regulation strategy use, and children's relational and physical aggression, in a Chinese sample. The findings document important associations between maternal emotional reappraisal and suppression and children's aggressive behaviors. The study makes an important contribution to the limited evidence base in this field, and suggests that further research is warranted to better understand the specific familial mechanisms involved in the development of childhood aggression. As knowledge grows in this area, more targeted and effective prevention and intervention strategies that aim to reduce aggressive behaviors in childhood can be developed.

Funding This work was supported by the Research Grant Council (Grant No.: GRF18607017).

\section{Declarations}

Conflict of Interest The authors declare that they have no conflict of interest.

Ethical Approval All procedures performed in studies involving human participants were in accordance with the ethical standards of the institutional and/or national research committee and with the 1964 Helsinki declaration and its later amendments or comparable ethical standards.

Open Access This article is licensed under a Creative Commons Attribution 4.0 International License, which permits use, sharing, adaptation, distribution and reproduction in any medium or format, as long as you give appropriate credit to the original author(s) and the source, provide a link to the Creative Commons licence, and indicate if changes were made. The images or other third party material in this article are included in the article's Creative Commons licence, unless indicated otherwise in a credit line to the material. If material is not included in the article's Creative Commons licence and your intended use is not permitted by statutory regulation or exceeds the permitted use, you will need to obtain permission directly from the copyright holder. To view a copy of this licence, visit http://creativecommons.org/licenses/by/4.0/.

\section{References}

1. Crick NR, Casas JF, Mosher M (1997) Relational and overt aggression in preschool. Dev Psychol 33(4):579-588. https:// doi.org/10.1037/0012-1649.33.4.579

2. Furniss T, Beyer T, Guggenmos J (2006) Prevalence of behavioural and emotional problems among six-years-old preschool children. Soc Psychiatry Psychiatr Epidemiol 41(5):394-399. https://doi.org/10.1007/s00127-006-0045-3

3. Evans SC, Frazer AL, Blossom JB, Fite PJ (2018) Forms and functions of aggression in early childhood. J Clin Child Adolesc Psychol 48(5):790-798. https://doi.org/10.1080/15374416. 2018.1485104
4. Crick NR, Grotpeter JK (1995) Relational aggression, gender, and social-psychological adjustment. Child Dev 66(3):710-722. https://doi.org/10.2307/1131945

5. Card NA, Stucky BD, Sawalani GM, Little TD (2008) Direct and indirect aggression during childhood and adolescence: a meta-analytic review of gender differences, intercorrelations, and relations to maladjustment. Child Dev 79(5):1185-1229. https://doi.org/10.1111/j.1467-8624.2008.01184.x

6. Bariola E, Hughes EK, Gullone E (2012) Relationships between parent and child emotion regulation strategy use: a brief report. J Child Fam Stud 21(3):443-448. https://doi.org/10.1007/ s10826-011-9497-5

7. Darling N, Steinberg L (1993) Parenting style as context: an integrative model. Psychol Bull 113(3):487-496. https://doi.org/ 10.1037/0033-2909.113.3.487

8. Pederson CA, Rathert JL, Fite PJ, Stoppelbein L, Greening L (2016) The role of parenting styles in the relation between functions of aggression and internalizing symptoms in a child psychiatric inpatient population. Child Psychiatry Hum Dev 47(5):819829. https://doi.org/10.1007/s10578-015-0614-3

9. Yap MB, Allen NB, Ladouceur CD (2008) Maternal socialization of positive affect: the impact of invalidation on adolescent emotion regulation and depressive symptomatology. Child Dev 79(5):1415-1431. https://doi.org/10.1111/j.1467-8624.2008. 01196.x

10. Nelson DA, Yang C, Coyne SM, Olsen JA, Hart CH (2013) Parental psychological control dimensions: connections with Russian preschoolers' physical and relational aggression. J Appl Dev Psychol 34(1):1-8. https://doi.org/10.1016/j.appdev.2012.07.003

11. Denham SA, Mitchell-Copeland J, Strandberg K, Auerbach S, Blair K (1997) Parental contributions to preschoolers' emotional competence: direct and indirect effects. Motiv Emot 21(1):65-86. https://doi.org/10.1023/A:1024426431247

12. Ramsden SR, Hubbard JA (2002) Family expressiveness and parental emotion coaching: their role in children's emotion regulation and aggression. J Abnorm Child Psychol 30(6):657-667. https://doi.org/10.1023/a:1020819915881

13. Williams SR, Woodruff-Borden J (2015) Parent emotion socialization practices and child self-regulation as predictors of child anxiety: the mediating role of cardiac variability. Child Psychiatry Hum Dev 46(4):512-522. https://doi.org/10.1007/ s10578-014-0492-0

14. Crespo LM, Trentacosta CJ, Aikins D, Wargo-Aikins J (2017) Maternal emotion regulation and children's behavior problems: the mediating role of child emotion regulation. J Child Fam Stud 26(10):2797-2809. https://doi.org/10.1007/s10826-017-0791-8

15. Xiao SX, Spinrad TL, Carter DB (2018) Parental emotion regulation and preschoolers' prosocial behavior: the mediating roles of parental warmth and inductive discipline. J Genet Psychol 179(5):246-255. https://doi.org/10.1080/00221325.2018.1495611

16. Zimmer-Gembeck MJ, Kerin JL, Webb HJ, Gardner AA, Campbell SM, Swan K et al (2019) Improved perceptions of emotion regulation and reflective functioning in parents: two additional positive outcomes of parent-child interaction therapy. Behav Ther 50:340-352. https://doi.org/10.1016/j.beth.2018.07.002

17. Thompson RA (1991) Emotional regulation and emotional development. Educ Psychol Rev 3(4):269-307. https://doi.org/10.1007/ BF01319934

18. Aldao A, Nolen-Hoeksema S, Schweizer S (2010) Emotion-regulation strategies across psychopathology: a meta-analytic review. Clin Psychol Rev 30(2):217-237. https://doi.org/10.1016/j.cpr. 2009.11.004

19. Gonçalves SF, Chaplin TM, Turpyn CC, Niehaus CE, Curby TW, Sinha R, Ansell EB (2019) Difficulties in emotion regulation predict depressive symptom trajectory from early to middle 
adolescence. Child Psychiatry Hum Dev 50(4):618-630. https:// doi.org/10.1007/s10578-019-00867-8

20. Gross JJ (1998) Antecedent- and response-focused emotion regulation: divergent consequences for experience, expression, and physiology. J Pers Soc Psychol 74(1):224-237. https://doi.org/ 10.1037//0022-3514.74.1.224

21. John OP, Gross JJ (2004) Healthy and unhealthy emotion regulation: personality processes, individual differences, and life span development. J Personal Psychol 72(6):1301-1334. https://doi. org/10.1111/j.1467-6494.2004.00298.x

22. Eisenberg N, Cumberland A, Spinrad TL (1998) Parental socialization of emotion. Psychol Inq 9(4):241-273. https://doi.org/10. 1207/s15327965pli0904_1

23. Lorber MF, Del Vecchio T, Feder MA, Slep AMS (2017) A psychometric evaluation of the revised Parental Emotion Regulation Inventory. J Child Fam Stud 26(2):452-463. https://doi.org/10. 1007/s10826-016-0578-3

24. Bridgett DJ, Gartstein MA, Putnam SP, Lance KO, Iddins E, Waits R et al (2011) Emerging effortful control in toddlerhood: the role of infant orienting/regulation, maternal effortful control, and maternal time spent in caregiving activities. Infant Behav Dev 34(1):189-199. https://doi.org/10.1016/j.infbeh.2010.12.008

25. Deater-Deckard K, Sewell MD, Petrill SA, Thompson LA (2010) Maternal working memory and reactive negativity in parenting. Psychol Sci 21(1):75-79

26. Edwards ES, Holzman JB, Burt NM, Rutherford HJV, Mayes LC, Bridgett DJ (2017) Maternal emotion regulation strategies, internalizing problems and infant negative affect. J Appl Dev Psychol 48:59-68. https://doi.org/10.1016/j.appdev.2016.12.001

27. Bandura A (1977) Social learning theory. Prentice Hall, Englewood Cliffs, NJ

28. Parke RD, Ladd GW (eds) (1992) Family-peer relationships: modes of linkage. Erlbaum, Hillsdale, NJ

29. Are F, Shaffer A (2016) Family emotion expressiveness mediates the relations between maternal emotion regulation and child emotion regulation. Child Psychiatry Hum Dev 47(5):708-715. https://doi.org/10.1007/s10578-015-0605-4

30. Morris AS, Silk JS, Steinberg L, Myers SS, Robinson LR (2007) The role of the family context in the development of emotion regulation. Soc Dev 16(2):361-388

31. Eisenberg N, Cumberland A, Spinrad TL, Fabes RA, Shepard SA, Reiser M et al (2001) The relations of regulation and emotionality to children's externalizing and internalizing problem behavior. Child Dev 72(4):1112-1134. https://doi.org/10.1111/1467-8624. 00337

32. Trentacosta CJ, Shaw DS (2009) Emotional self-regulation, peer rejection, and antisocial behavior: developmental associations from early childhood to early adolescence. J Appl Dev Psychol 30(3):356-365. https://doi.org/10.1016/j.appdev.2008.12.016

33. Coplan RJ, Hastings PD, Lagacé-Séguin DG, Moulton CE (2002) Authoritative and authoritarian mothers' parenting goals, attributions, and emotions across different childrearing contexts. Parent Sci Pract 2(1):1-26. https://doi.org/10.1207/S15327922P AR0201 1

34. Denson TF, DeWall CN, Finkel EJ (2012) Self-control and aggression. Curr Dir Psychol Sci 21(1):20-25

35. Roberts WL (1999) The socialization of emotional expression: relations with prosocial behaviour and competence in five samples. Can J Behav Sci/Rev Can Sci Comport 31(2):72-85. https:// doi.org/10.1037/h0087075

36. Kim B, Teti DM, Cole PM (2012) Mothers' affect dysregulation, depressive symptoms, and emotional availability during motherinfant interaction. Infant Ment Health J 33(5):469-476. https:// doi.org/10.1002/imhj. 21326

37. Zimmer-Gembeck MJ, Thomas R (2010) Parents, parenting and toddler adaptation: evidence from a national longitudinal study of
Australian children. Infant Behav Dev 33(4):518-529. https://doi. org/10.1016/j.infbeh.2010.07.004

38. Berndt TJ, Cheung PC, Lau S, Hau KT, Lew WJ (1993) Perceptions of parenting in mainland China, Taiwan, and Hong Kong: sex differences and societal differences. Dev Psychol 29(1):156164. https://doi.org/10.1037/0012-1649.29.1.156

39. Ma JL, Wong TK, Lau YK (2009) Sex differences in perceived family functioning and family resources in Hong Kong families: implications for social work practice. Asian Soc Work Policy Rev 3(3):155-174. https://doi.org/10.1111/j.1753-1411.2009.00031.x

40. McHale JP, Rao N, Krasnow AD (2000) Constructing family climates: chinese mothers' reports of their co-parenting behaviour and preschoolers' adaptation. Int J Behav Dev 24(1):111-118

41. Chen X, Dong Q, Zhou H (1997) Authoritative and authoritarian parenting practices and social and school performance in Chinese children. Int J Behav Dev 21(4):855-873

42. Lau EYH (2016) A mixed-methods study of paternal involvement in Hong Kong. Br Educ Res J 42(6):1023-1040. https://doi.org/ 10.1002/berj. 3248

43. Kim SW, Hill NE (2015) Including fathers in the picture: a metaanalysis of parental involvement and students' academic achievement. J Educ Psychol 107(4):919-934. https://doi.org/10.1037/ edu0000023

44. Harvey E, Danforth JS, Eberhardt Mcgee T, Ulaszek WR, Friedman JL (2003) Parenting of children with attention-deficit/hyperactivity disorder (ADHD): the role of parental ADHD symptomatology. J Atten Disord 7(1):31-42. https://doi.org/10.1177/10870 5470300700104

45. Martins EC, Soares I, Martins C, Osório A (2016) Infants's style of emotion regulation with their mothers and fathers: concordance between parents and the contribution of father-infant interaction quality. Soc Dev 25(4):812-827. https://doi.org/10.1111/sode. 12171

46. Gallegos MI, Murphy SE, Benner AD, Jacobvitz DB, Hazen NL (2017) Marital, parental, and whole-family predictors of toddlers' emotion regulation: the role of parental emotional withdrawal. J Fam Psychol 31(3):294-303. https://doi.org/10.1037/fam0000245

47. Wang Y, Wang M, Xing X (2018) Parental harsh discipline and child emotion regulation: the moderating role of parental warmth in China. Child Youth Serv Rev 93:283-290. https://doi.org/10. 1016/j.childyouth.2018.07.035

48. Rodriguez CM, Baker LR, Pu DF, Tucker MC (2017) Predicting parent-child aggression risk in mothers and fathers: role of emotion regulation and frustration tolerance. J Child Fam Stud 26(9):2529-2538. https://doi.org/10.1007/s10826-017-0764-y

49. Cassano M, Perry-Parrish C, Zeman J (2007) Influence of gender on parental socialization of children's sadness regulation. Soc Dev 16(2):210-231. https://doi.org/10.1111/j.1467-9507.2007.00381.x

50. Chen X, Wu X, Wang Y (2018) Mothers' emotional expression and discipline and preschoolers' emotional regulation strategies: gender differences. J Child Fam Stud 27:3709-3716. https://doi. org/10.1007/s10826-018-1199-9

51. Fivush R, Brotman MA, Buckner JP, Goodman SH (2000) Gender differences in parent-child emotion narratives. Sex Roles 42(3/4):233-253. https://doi.org/10.1023/A:1007091207068

52. Rogers CE, Anderson PJ, Thompson DK, Kidokoro H, Wallendorf M, Treyvaud K et al (2012) Regional cerebral development at term relates to school-age social-emotional development in very preterm children. J Am Acad Child Adolesc Psychiatr 51(2):181191. https://doi.org/10.1016/j.jaac.2011.11.009

53. Miner JL, Clarke-Stewart KA (2008) Trajectories of externalizing behavior from age 2 to age 9: relations with gender, temperament, ethnicity, parenting, and rater. Dev Psychol 44(3):771-786. https://doi.org/10.1037/0012-1649.44.3.771

54. Stephan WG, Stephan CW, De Vargas MC (1996) Emotional expression in Costa Rica and the United States. J Cross Cult 
Psychol 27(2):147-160. https://doi.org/10.1177/0022022196 272001

55. Butler EA, Lee TL, Gross JJ (2007) Emotion regulation and culture: are the social consequences of emotion suppression culturespecific? Emotion 7(1):30-48. https://doi.org/10.1037/15283542.7.1.30

56. Cheung RY, Park IJ (2010) Anger suppression, interdependent self-construal, and depression among Asian American and European American college students. Cultur Divers Ethnic Minor Psychol 16(4):517-525

57. Yuan J, Liu Y, Ding N, Yang J (2014) The regulation of induced depression during a frustrating situation: benefits of expressive suppression in Chinese individuals. PLoS ONE 9(5):1-11. https:// doi.org/10.1371/journal.pone.0097420

58. Soto JA, Perez CR, Kim Y, Lee EA, Minnick MR (2011) Is expressive suppression always associated with poorer psychological functioning? A cross-cultural comparison between European Americans and Hong Kong Chinese. Emotion 11(6):1450-1455. https://doi.org/10.1037/a0023340

59. Cheung RY, Leung MC, Chan KK, Lam CB (2019) Effects of mother-offspring and father-offspring dynamics on emerging adults' adjustment: the mediating role of emotion regulation. PLoS ONE 14(2):1-16. https://doi.org/10.1371/journal.pone. 0212331

60. Roberts NA, Levenson RW, Gross JJ (2008) Cardiovascular costs of emotion suppression cross ethnic lines. Int J Psychophysiol 70(1):82-87. https://doi.org/10.1016/j.ijpsycho.2008.06.003

61. Lamb ME (1987) The emergent American father. In: Lamb ME (ed) The father's role: cross-cultural perspectives. Lawrence Erlbaum, Hillsdale, NJ, pp 3-26

62. Marsiglio W, Amato P, Day RD, Lamb ME (2000) Scholarship on fatherhood in the 1990s and beyond. J Marriage Fam 62(4):11731191. https://doi.org/10.1111/j.1741-3737.2000.01173.x

63. Pedro MF, Ribeiro T, Shelton KH (2012) Marital satisfaction and partners' parenting practices: the mediating role of coparenting behavior. J Fam Psychol 26(4):509-522. https://doi.org/10.1037/ a0029121

64. Hong Kong Census and Statistics Department (2016) 2016 population by-census: thematic report household income distribution in Hong Kong. https://www.bycensus2016.gov.hk/data/16bc-house hold-income.pdf. Accessed 19 Nov 2020

65. Gross JJ, John OP (2003) Individual differences in two emotion regulation processes: implications for affect, relationships, and well-being. J Pers Soc Psychol 85(2):348-362. https://doi.org/10. 1037/0022-3514.85.2.348

66. Crick NR, Casas JF, Ku H (1999) Relational and physical forms of peer victimization in preschool. Dev Psychol 35(2):376-385. https://doi.org/10.1037//0012-1649.35.2.376

67. Muthén LK, Muthén BO (1998-2012) MPlus users guide, 6th edn. Muthén Muthén, Los Angeles

68. Rutherford HJ, Wallace NS, Laurent HK, Mayes LC (2015) Emotion regulation in parenthood. Dev Rev 36:1-14. https://doi.org/ 10.1016/j.dr.2014.12.008

69. McDowell DJ, Kim M, O'neilParke RRD (2002) Children's emotional regulation and social competence in middle childhood.
Marriage Fam Rev 34(3-4):345-364. https://doi.org/10.1300/ J002v34n03_07

70. McElwain NL, Halberstadt AG, Volling BL (2007) Mother- and father-reported reactions to children's negative emotions: relations to young children's emotional understanding and friendship quality. Child Dev 78(5):1407-1425. https://doi.org/10.1111/j. 1467-8624.2007.01074.x

71. Gottman JM, Katz LF, Hooven C (1996) Parental meta-emotion philosophy and the emotional life of families: theoretical models and preliminary data. J Fam Psychol 10(3):243-268. https://doi. org/10.1037/0893-3200.10.3.243

72. Kienhuis M, Rogers S, Giallo R, Matthews J, Treyvaud K (2010) A proposed model for the impact of parental fatigue on parenting adaptability and child development. J Reprod Infant Psychol 28(4):392-402. https://doi.org/10.1080/02646830903487383

73. Morris AS, Silk JS, Morris MD, Steinberg L, Aucoin KJ, Keyes AW (2011) The influence of mother-child emotion regulation strategies on children's expression of anger and sadness. Dev Psychol 47(1):213-225. https://doi.org/10.1037/a0021021

74. Ehring T, Tuschen-Caffier B, Schnülle J, Fischer S, Gross JJ (2010) Emotion regulation and vulnerability to depression: spontaneous versus instructed use of emotion suppression and reappraisal. Emotion 10(4):563-572. https://doi.org/10.1037/a0019 010

75. Haga SM, Kraft P, Corby E (2009) Emotion regulation: antecedents and well-being outcomes of cognitive reappraisal and expressive suppression in cross-cultural samples. J Happiness Stud 10(3):271-291. https://doi.org/10.1007/s10902-007-9080-3

76. Karnilowicz HR, Waters SF, Mendes WB (2018) Not in front of the kids: effects of parental suppression on socialization behaviors during cooperative parent-child interactions. Emotion 19(7):1183-1191. https://doi.org/10.1037/emo0000527

77. Song JH, Trommsdorff G (2016) Linking maternal emotion socialization to boys' and girls' emotion regulation in Korea. Child Stud Asia Pac Context 6:47-57. https://doi.org/10.5723/csac.2016.6.2. 047

78. Havighurst SS, Wilson KR, Harley AE, Kehoe C, Efron D, Prior MR (2013) “Tuning into Kids": reducing young children's behavior problems using an emotion coaching parenting program. Child Psychiatry Hum Dev 44(2):247-264. https://doi.org/10.1007/ s10578-012-0322-1

79. Juliano M, Werner RS, Cassidy KW (2006) Early correlates of preschool aggressive behavior according to type of aggression and measurement. J Appl Dev Psychol 27(5):395-410. https://doi.org/ 10.1016/j.appdev.2006.06.008

80. Lau EYH (2017) Parenting and childhood aggression in the Chinese context: an examination of parental responses, physical coercion and warmth. Early Years 39(1):1-15. https://doi.org/10.1080/ 09575146.2017 .1344195

Publisher's Note Springer Nature remains neutral with regard to jurisdictional claims in published maps and institutional affiliations. 